Ann. Zootech., I975, 24 (4), 757-763.

NOTE

\title{
INFLUENCE COMPARÉE DE LA MACHINE DE RÉCOLTE SUR LES QUANTITÉS D'ENSILAGE INGÉRÉES PAR DES GÉNISSES ET DES MOUTONS
}

\author{
J. P. DULPHY et Brigitte MICHALET \\ avec la collabotation technique de J. P. Andrieu, J. M. Borssay, \\ H. Borsquit et L. L'Hotelier \\ Laboratoire des Aliments, \\ Cen de Recherches de Clermont-Ferrand, I. N.R. A., \\ Theix, Saint Genès Champanelle, 63110 Beaumont
}

\section{RÉSUMÉ}

Nous avons comparé l'influence de la machine de récolte sur les quantités d'ensilage ingérées par des génisses de race laitière et par des moutons. Pour cela, nous avons, à partir de 3 fourrages verts dont on mesurait les quantités ingérées par des génisses et des moutons, préparé 6 ensilages (3 à brins courts, 3 à brins longs).

Sur la base de la matière sèche, les quantités d'ensilage ingérées ont représenté pour les génisses 93,3 p. Ioo des quantités ingérées avec les fourrages verts correspondants et pour les moutons seulement 60,2 p. Ioo.

Les ensilages à brins courts ont été ingérés en quantité plus importante que les ensilages à brins longs : l'accroissement a été respectivement de $+93,6 \mathrm{p}$. Ioo et $+32,9$ p. Ioo en moyenne chez les moutons et chez les génisses.

Deux autres essais avaient été effectués par ailleurs à Orcival (DULPHy et DemarguiLLy, I 975 a). Dans ces deux cssais, les augmentations de quantités ingérées avaient été plus faibles : respectivement de $+28,5 \mathrm{p}$. Ioo et de $7,0 \mathrm{p}$. Ioo.

L'ensemble de ces 5 essais montre que, d'une part, l'ensilage entraîne, par comparaison au fourrage vert sur pied correspondant une diminution d'ingestibilité plus importante chez le Mouton que chez la Génisse et que, d'autre part, le Mouton est plus sensible que la Génisse à la finesse de hachage des ensilages.

En regroupant tous ces résultats, et en faisant abstraction de la machine de récolte, on peut calculer une relation simple entre les quantités de matière sèche d'ensilage ingérées par les génisses $\left(\mathrm{Y}\right.$ en $\mathrm{g} / \mathrm{kg} \mathrm{P}^{0,75}$ ) et celles ingérées par les moutons $(\mathrm{X})$ :

$$
\mathrm{Y}={ }_{44}, 6+0,83 \mathrm{X} \quad\left(r=0,959^{* *} ; n=\mathrm{I} 0\right)
$$




\section{INTRODUCTION}

La finesse de hachage des ensilages est un facteur primordial de leur ingestibilité. Les ensilages à brins courts ont été ingérés par les moutons en quantité beaucoup plus élevée (plus de $40 \mathrm{p}$. IOo) que les ensilages à brins longs correspondants dans les essais de DULPhy et Demarquiriy (r973). L'accroissement est cependant beaucoup plus faible chez les bovins puisqu'il n'a été que de $6,4 \mathrm{p}$. Ioo chez des génisses de I an (Dulphy et Demarquiliy, I975 a) et de I7,8 p. Ioo chez des vaches laitières (DULPHY et DEMARQUILLY, I975 $b$ ).

Les résultats sur bovins ayant été obtenus sur d'autres ensilages que ceux testés sur moutons et dans des conditions différentes (les génisses et les vaches laitières recevaient en plus de l'ensilage offert à volonté de l'aliment concentré alors que les moutons n'en recevaient pas), nous avons préparé de nouveaux ensilages à brins courts ou longs et les avons distribués simultanément et dans des conditions rigoureusement comparables à des génisses de I an et à des moutons. Pour mieux préciser l'influence de la technique d'ensilage et de la finesse de hachage, l'ingestibilité des fourrages verts initiaux avait été mesurée avec des animaux comparables. En regroupant les résultats de ces nouveaux essais avec ceux obtenus lors des essais précédents (Dulphy et Demarquilly, I975 a), nous avons en outre étudié s'il existait une liaison entre les quantités d'ensilage ingérées par les moutons et celles ingérées par les génisses et si cette liaison dépendait ou non de la finesse de hachage des ensilages.

\section{MATÉRIFL, FT MÉTHODES}

A partir de 3 fourrages verts cultivés à Theix (un ray-grass anglais Réveille au début de l'épiaison, une fétuque élevée Ludelle à l'épiaison et une repousse de $2^{\circ}$ cycle âgée de 6 semaines d'un ray-grass italien Tétrone), nous avons préparé pour chacun d'eux deux ensilages, l'un à brins courts récolté avec une machine à tambour hacheur, l'autre à brins longs récolté avec une machine à fléaux. Les ensilages réalisés avec addition d'acide formique à 85 p. Ioo à la dose de 5 litres par tonne pour le ray-grass anglais et de 3,5 litres pour la fétuque et le ray-grass italien ont été préparés en petits silos tours (poches en Sainter) contenant de 7 à Io tonnes le fourrage frais. I.es caractéristiques des fourrages verts et des ensilages correspondants sont présentés au tableau $\mathrm{r}$.

Ces fourrages ont été distribués individuellement à volonté ( 10 p. I oo de rerus) comme seul aliment en 2 repas par jour (à 8 ct $\mathbf{1} 6$ h) à 5 génisses de I an (race Firisonne et Montbéliarde) en stabulation entravée, sans paille et à 6 moutons mâles castrés de 2 ans (race Texel) maintenus en cage à métabolisme. I es fourrages verts étaient fauchés et récoltés chaque matin puis hachés dans un hache-paille (brins d'environ $3 \mathrm{~cm}$ ). La période de mesure des quantités ingérées a été de 6 jours et était précédée d'une période pré-expérimentale de ro jours pour les fourrages verts et de $I+$ jours pour les ensilages. C'est au milieu de chaque période de mesure pour le fourrage vert qu'ont été préparés les différents ensilages étudiés. Les animaux utilisés pour les mesures sur fourrages verts durant l'été et sur les ensilages durant l'hiver suivant étaient choisis pour être aussi semblables que possible.

Les méthodes d'analyse des ensilages ont été rapportées précédemment (Dulphy et DemarQURLLY, I975 a).

Aux 3 essais réalisés à Theix nous en avons joint 2 réalisés à ()rcival (essais 2 et 3 de DULPHY 
COMPARAISON GÉNISSES-MOUTONS

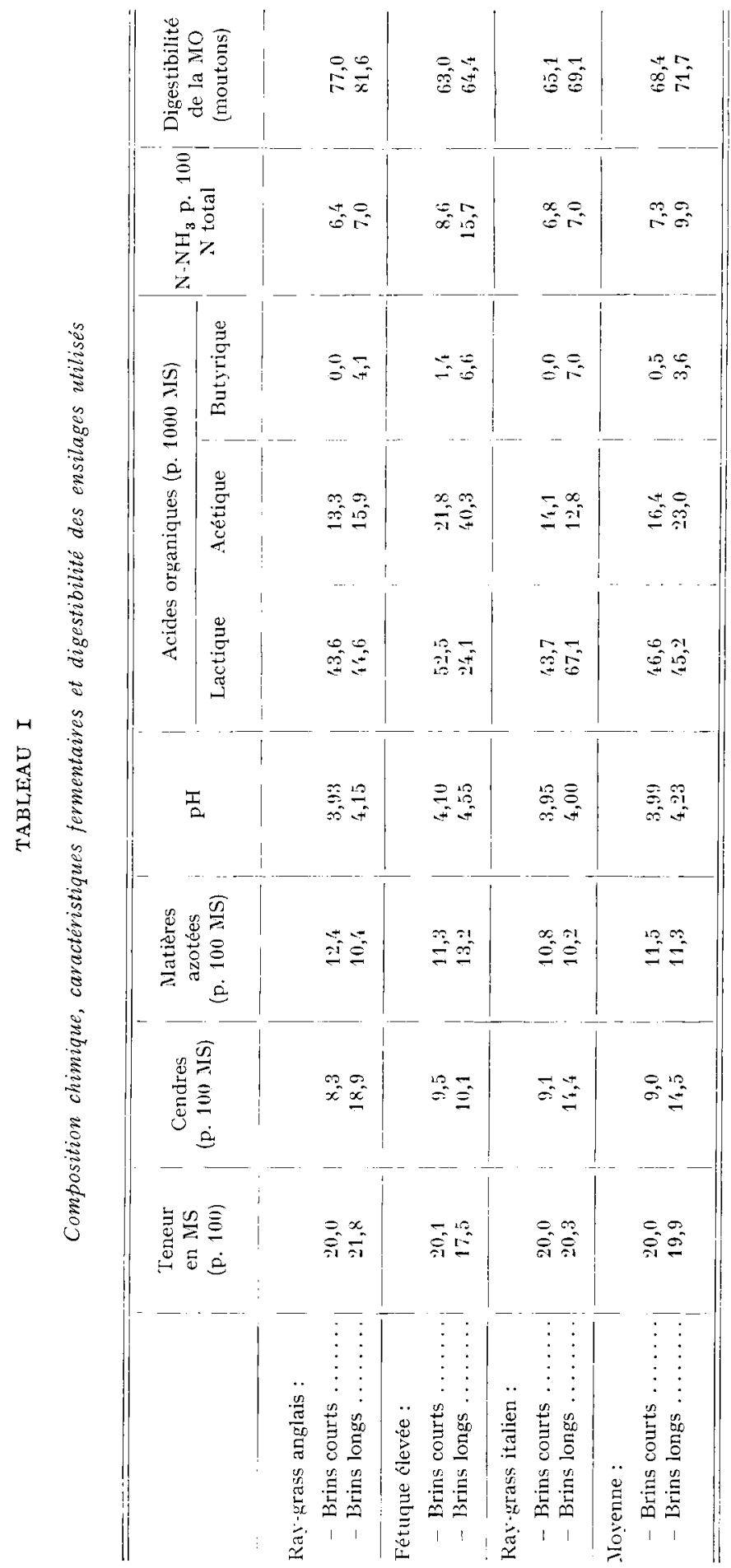


et Demarquilly, I975 a). Les génisses recevaient alors un aliment concentré distribué à deux niveaux. En extrapolant, il est possible d'estimer les quantités d'ensilage ingérées $\left(\mathrm{g} / \mathrm{kg} \mathrm{P}^{0,75}\right)$ en absence d'aliment concentré :

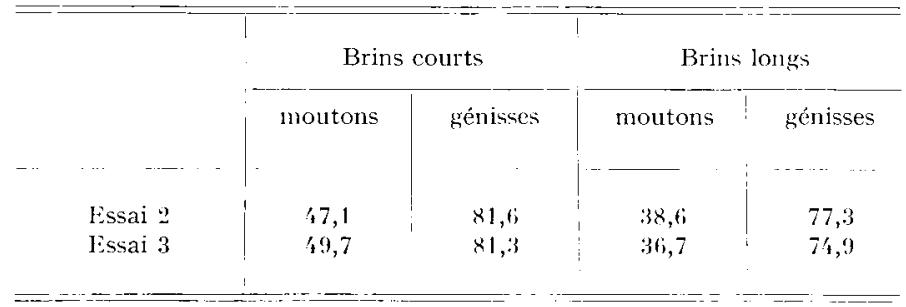

\section{RÉSULTATS ET DISCUSSION}

\section{Comparaison des quantités ingérées par les génisses et les moutons (tabl. 2)}

Par rapport aux quantités de fourrage vert ingérées, les quantités d'ensilage ingérées ont été en moyenne de 93,3 p. Ioo pour les génisses et seulement de 60,2 p. Ioo dans les mêmes conditions pour les moutons. Il semble donc que l'ensilage entraîne une diminution d'ingestibilité plus importante chez le Mouton que chez la Génisse. Nous essaierons de confirmer ces résultats qui montrent, par ailleurs, qu'11 est nécessaire de vérifier sur des bovins les résultats obtenus sur des moutons.

Les ensilages à brins courts, dans les 3 essais réalisés à Theix ont été ingérés en quantité plus importante que les ensilages à brins longs : respectivement $+93,6$ p. Ioo et $+32,9$ p. Ioo en moyenne pour les moutons et les génisses.

En valeur absolue, en $\mathrm{g} / \mathrm{kg} \mathrm{P}^{0,75}$, les augmentations de quantités ingérées entre ensilage à brins longs et ensilage à brins courts ont été comparables chez les moutons et chez les génisses : +22 et $+2 \mathrm{I} \mathrm{g}$. Notons aussi que la quantité ingérée a beaucoup moins varié d'un ensilage à l'autre lorsque ces ensilages étaient à brins longs que lorsqu'ils étaient à brins courts. Ceci est particulièrement net chez les moutons, mais est également vrai chez les génisses. Chez ces deux catégories d'animaux la quantité d'ensilage à brins longs ingérée est donc réglée par d'autres facteurs que l'ingestibilité du fourrage vert initial et la qualité de conservation de l'ensilage.

L'influence de la machine de récolte sur les quantités d'ensilage ingérées par les génisses est donc beaucoup plus importante dans les essais réalisés à Theix que dans ceux réalisés à Orcival (DEMARQUILLY et DulPhy, I975 a). Dans ces derniers, nous avions trouvé des différences plus faibles, mais dans un cas, l'ensilaye à brins courts était mal conservé et dans l'autre les 2 ensilages étaient très bien conservés. Ces différences plus faibles ont peut-être été liées au tassement plus efficace effectué dans les silos d'Orcival (50 à 60 tonnes de fourrage) que dans ceux de The1x (7 à ro tonnes). Fin revanche, dans les essa1s réalisés à Theix, les ensilages à brins longs ont été moins bien conservés que ceux à brins courts. A Theix, il est donc vraisemblable que les effets finesse de hachage et qualité de conservation se soient conjugués pour augmenter la différence entre les quantités ingérées des 2 types d'ensilage par les génisses. 
TABLEAU 2. -- Quantités de matière sèche ingérées par les montons et les génisses

\begin{tabular}{|c|c|c|c|c|c|c|}
\hline & \multicolumn{3}{|c|}{ Moutons } & \multicolumn{3}{|c|}{ Génisses } \\
\hline & \multirow[b]{2}{*}{$\begin{array}{c}\text { Poids } \\
\text { moyen (kg) }\end{array}$} & \multicolumn{2}{|c|}{ Matière sèche ingćrée } & \multirow[b]{2}{*}{$\begin{array}{c}\text { Poids } \\
\text { moyen }(\mathrm{kg})\end{array}$} & \multicolumn{2}{|c|}{ Matière sèche ingérée } \\
\hline & & $\begin{array}{c}\text { en } g / k g \\
\mathrm{P}^{\mathbf{0}, 75}\end{array}$ & $\begin{array}{c}\text { en } \\
\mathrm{kg} / 100 \mathrm{~kg} \\
\mathrm{PV}\end{array}$ & & $\begin{array}{c}\text { en } g / k g \\
P^{0,75}\end{array}$ & $\begin{array}{c}\text { en } \\
\mathrm{kg} / 100 \mathrm{~kg} \\
\mathrm{PV}\end{array}$ \\
\hline \multicolumn{7}{|l|}{ Ray-grass anglais : } \\
\hline - Fourrage vert ........ & 60 & 67,9 & 2,45 & 315 & 85,3 & 2,02 \\
\hline - Ensilage brins courts ... & 66 & 54,2 & 1,91 & 300 & 91,7 & 2,20 \\
\hline - Ensilage brins longs..... & 64 & $2 \prime^{\prime}, 8$ & 0,88 & 305 & 68,5 & 1,65 \\
\hline \multicolumn{7}{|l|}{ Fétuque élevée : } \\
\hline - Fourrage vert $\ldots \ldots$ & 64 & 42,9 & 1,52 & 345 & $6 ; 5,1$ & 1,51 \\
\hline - Ensilage brins courts & $5^{\prime} t$ & 34,7 & 1,28 & 329 & 75,7 & 1,78 \\
\hline - Ensilage brins longs..... & 51 & 23,2 & 0,87 & 332 & 59,8 & 1,40 \\
\hline \multicolumn{7}{|l|}{ Ray-grass italien : } \\
\hline - Fourrage vert $\ldots . . \ldots$ & 51 & 61,0 & 2,28 & 347 & 88,9 & 2,06 \\
\hline - Ensilage brins courts & 53 & 47,5 & 1,83 & 320 & 87,2 & 2,06 \\
\hline - Ensilage brins longs. .... & 51 & 22,6 & 0,85 & 304 & 63,4 & 1,52 \\
\hline \multicolumn{7}{|l|}{ Moyenne: } \\
\hline - Fourrage vert $\ldots \ldots \ldots$ & 58 & 57,3 & 2,08 & 336 & 79,7 & 1,95 \\
\hline - Ensilage brins courts ... & 58 & 45,5 & 1,67 & 316 & 84,9 & 2,01 \\
\hline - Ensilage brins longs..... & 55 & 23,5 & 0,87 & 314 & 63,9 & 1,52 \\
\hline
\end{tabular}

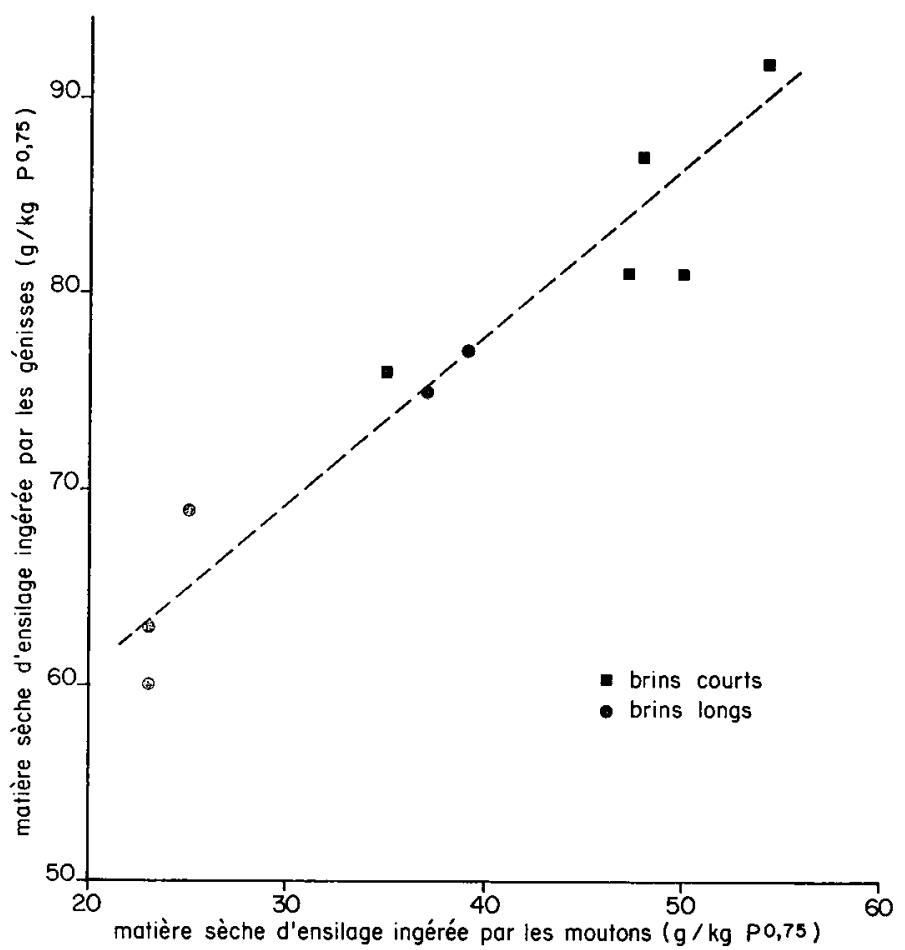

HIG. I. -- Relation entre les quantités d'ensilage ingérées par les moutons el les gênisses

Annales de Zootechnie. - 1975. 
Quoi qu'il en soit, ces nouveaux essais, confirmant les précédents, montrent que les moutons sont plus sensibles que les génisses à la finesse de hachage des ensilages ; l'augmentation de la quantité de matière sèche ingérée entraînée par le hachage fin far comfaraison au hachage grossier des ensilages a été de 58,o p. roo chez les moutons contre $2 \mathrm{I}, 4 \mathrm{p}$. Ioo chez les génisses, en moyenne pour les 5 essais, avec cependant une différence notable selon la plus ou moins grande différence de qualité de conservation entre l'ensilage à brins longs et celui à brins courts.

Les causes de la sensibilité plus grande des moutons, tant à la finesse de hachage qu'au mode de conservation par ensilage, sont encore mal connues mais semblent pouvoir s'expliquer par des différences dans le comportement alimentaire et mérycique de ces deux catégories d'animaux (Mrchales, en préparation).

\title{
Relation entre les quantités \\ ingérées par les moutons et les génisses (fig. I)
}

En regroupant les résultats de nos cinq essais, nous disposons donc des mesures de quantités ingérées pour ro ensilages. E,n faisant abstraction de la machine de récolte, nous pouvons calculer une relation simple entre les quantités ingérées (en $\left.\mathrm{g} / \mathrm{kg} \mathrm{P}^{0,{ }^{75}}\right)$ par les génisses $(\mathrm{Y})$ et celles ingérées par les moutons $(\mathrm{X})$ :

$$
\mathrm{Y}=44,6+0,83 \mathrm{X} \quad\left(r=0,959^{* * ;} n=\mathrm{IO}\right)
$$

Il existe donc une liaison très étroite entre les quantités d'ensilage ingérées par les moutons et celles ingérées par les génisses et il est possible de prévoir les quantités d'ensilage ingérées par les génisses à partir de celles ingérées par les moutons lorsqu'il n'y a aucun apport d'aliment concentré complémentaire dans la ration.

\section{Rę̧u pour publication en avril 1975.}

\section{SUMMARY}

\author{
COMPARISON OF THE AMOUNTS OF SILAGE INGESTED BY HEIFERS
}

AND SHEEP AS AFFECTED BY THE TYPE OF FORAGE HARVESTER

The influence of the type of harvester on the silage intake level was compared in dairy heifers and sheep. For that purpose, 6 silages ( 3 finely chopped, 3 coarsely chopped ones, table I) were prepared from 3 green forages, the intake level of which was measured on heifers and sheep.

As compared to the amount of green forage dry matter ingested, the intake of silage dry matter represented 93.3 p. Ioo with the heifers and only $60.2 \mathrm{p}$. Ioo under the same conditions, with the sheep.

The intake of finely chopped silages was higher than that of coarsely chopped ones, i.e. on an average +93.6 p. Ioo and +32.9 p. Ioo, respectively for sheep and heifers (table 2).

In two other trials carricd out at Orcival (DULPHY and DEMARquILLY, I975 a), the increases recorded in the intake of finely chopped silages as compared to the coarsely chopped ones were lower : +28.5 p. Ioo and 7 . oo, respectively.

Taken as a whole, these 5 trials show, on the one hand, that the voluntary intake of silage, as compared to that of the same original green forage, is more reduced with the sheep than with heifers and, on the other hand, that sheep are more susceptible than heifers to the chopping fineness of the silages. 
By regrouping all these findings and by leaving out of account the type of forage harvester, a simple relation can be calculated between the amounts of silage dry matter ingested by the heifers $\left(\mathrm{Y}\right.$ in $\mathrm{g} / \mathrm{kg} \mathrm{P}^{0,75}$ ) and those ingested by the sheep $(\mathrm{X})$.

$$
\mathrm{Y}=44.6+0.83 \mathrm{X} \quad\left(r=0.959^{* *} ; n=\right.\text { Io) (fig. I). }
$$

\section{RÉFÉRENCES BIBLIOGRAPHIQUES}

Dulphy J. P., Demarquilly C., I973. Influence de la machine de récolte et de la finesse de hachage sur la valeur alimentaire des ensilages. Ann. Zootech., 22, 199-2 I7.

Dulphy J. P., Demarguilly C., I975 a. Influence de la machine de récolte sur la valeur des ensilages de graminées pour les génisses de race laitière. Ann. Zootech., 24, 35I-362.

Dulphy J. P., Demaroully C., I975 b. Influence de la machine de récolte sur les quantités d'ensilage ingérées et les performances des vaches laitières. Ann. Zootech., 24, 363-37I.

Michalet B., 1975. Thèse de Dr Ing. sur les facteurs de variation des quantités d'ensilage ingérées (en préparation). 